\title{
دراسة اقتصادية للتجارة الخارجية للقلقل المصري
}

\section{إنجى أحمد طعيمة ، إيمان عبد العزيز إبراهيم}

باحث بمعهز بحوث الإقتصاد الزراعي ـ مركز البحوث الزراعية

الملخص: على الرغم من اتجاه الدولة في السنوات الأخيرة إلى التوسع في الصادرات الزراعية من محاصيل

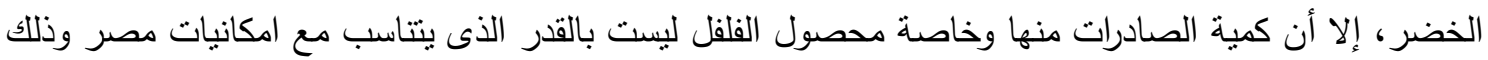

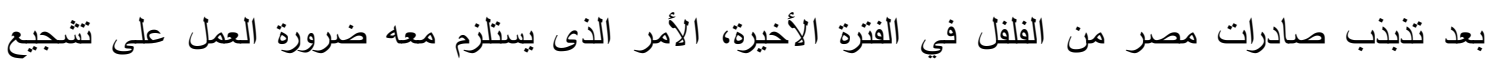

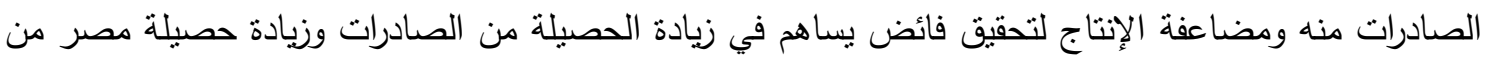

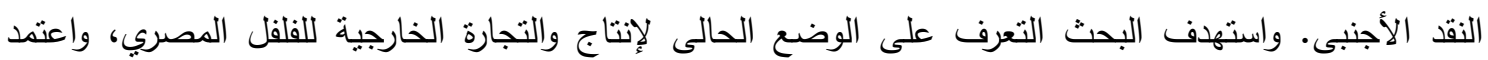
البحث في تحقيق أهدافه على كل من استخدام الأسلوب التحليلى الوصفي والقياسى، استخدام تحليل الإنحدار

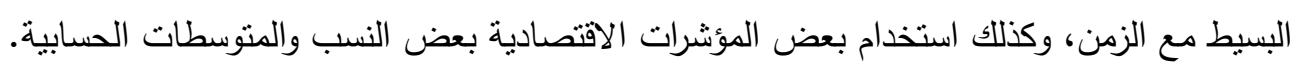

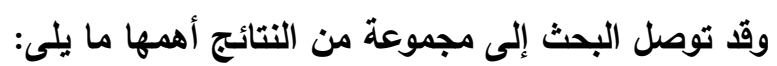

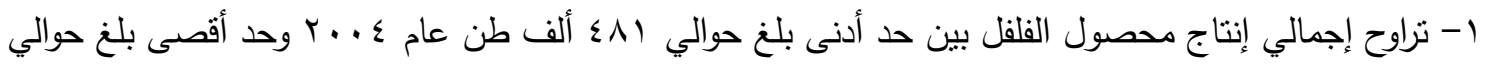

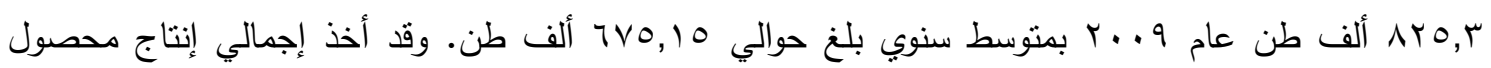

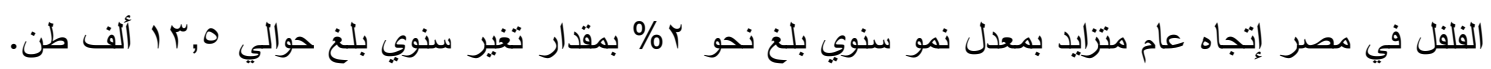

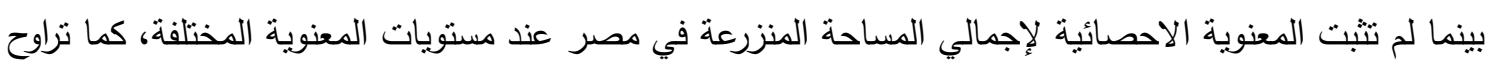

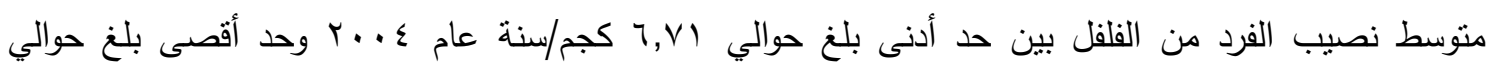

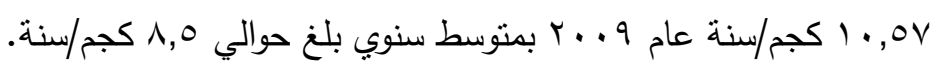

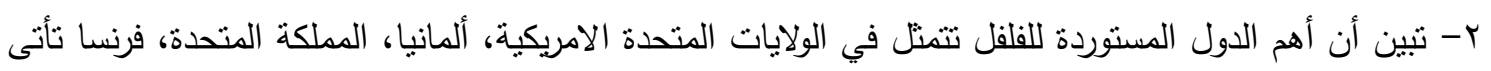

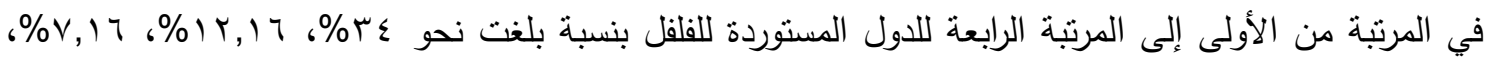

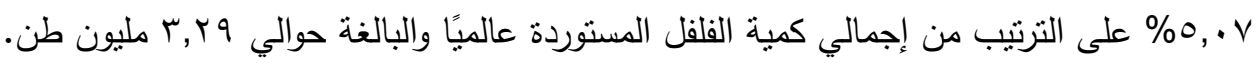

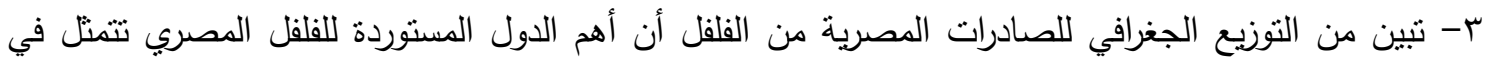

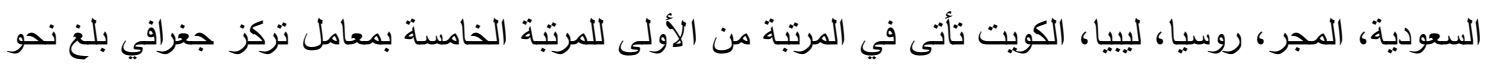

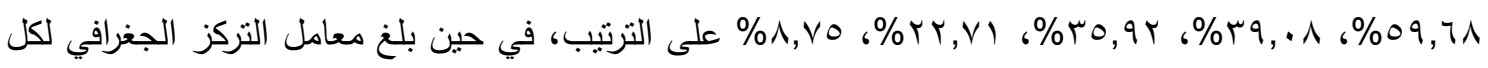

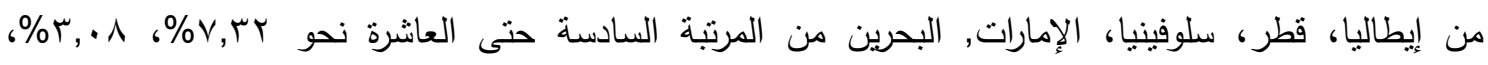

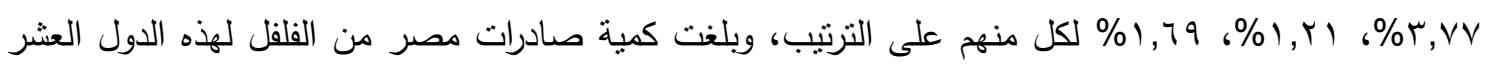

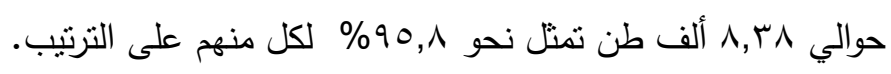

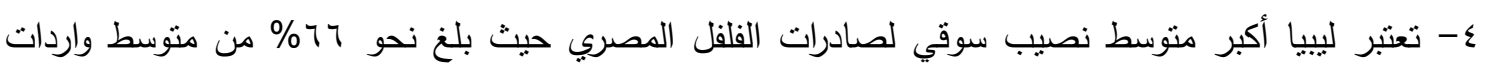

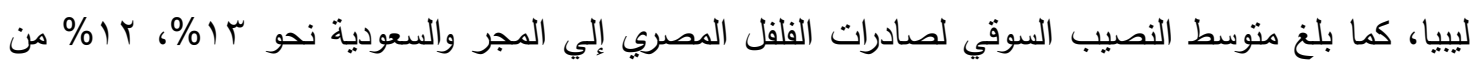

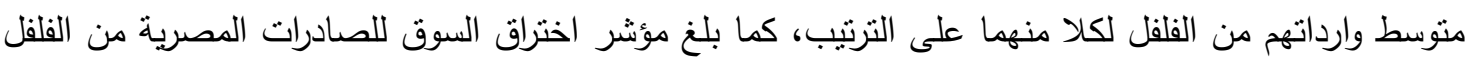

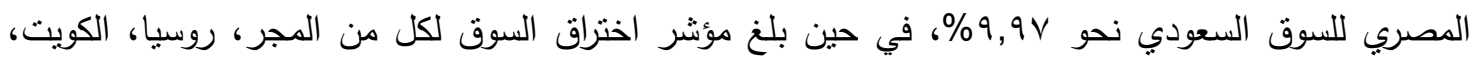

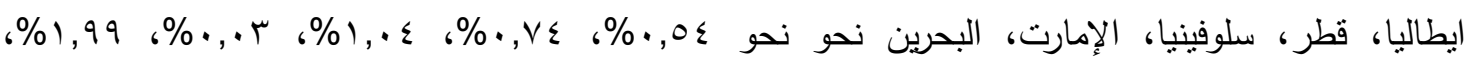




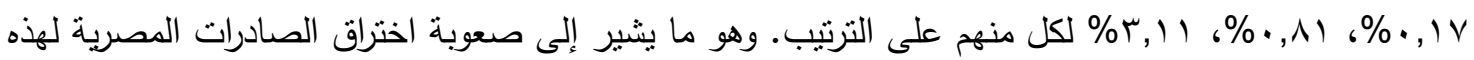
الأسواق. يتمتع الفلفل المصري بميزة تنافسية سعرية بالسوق السعودي مقارنة بمتوسط سعر تصدير بنجلاديش،

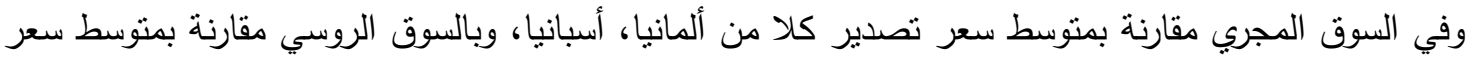
تصدير إسرائيل، كما نبين عدم وجود ميزة نسبية سعرية في كل من الكويت، قطر ، سلوفينيا، الأمارات، البحرين مقارنة بأسعار الدول المنافسة.

الكلمات المفتاحية: التجارة الخارجية الزراعية، الفلفل المصري، مؤشرات القدرة التتافسية، النصيب السوقي، معامل التركز الجغرافي، التتافسية السعرية.

\section{المقدمة}

تُعد تتمية الصادرات الزراعية من القضايا الهامة التي تحتل مكانة بارزة على خريطة أولويات السياسة الاقتصادية المصرية لكونها أحد مصادر النقد الأجنبي في ظل العجز المتزايد في الميزان التجاري الزراعي المصري والبالغ

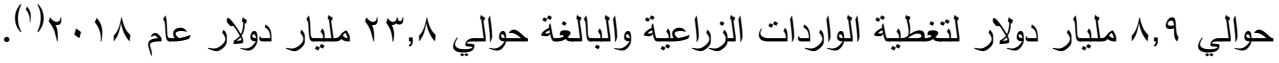

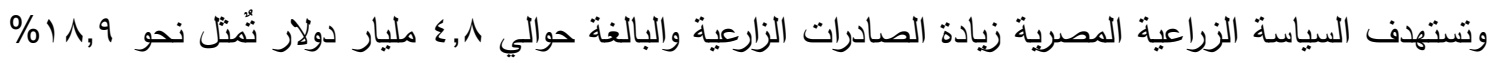
من إجمالي الصادرات الكلية المصرية.وتحتل الصادرات الزراعية من الخضر أهمية كبيرة في الصادرات الزراعية

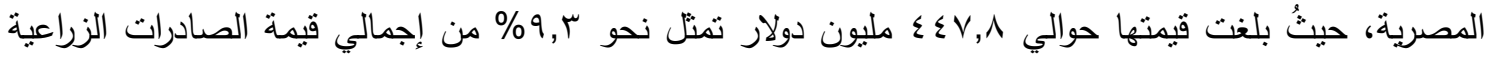

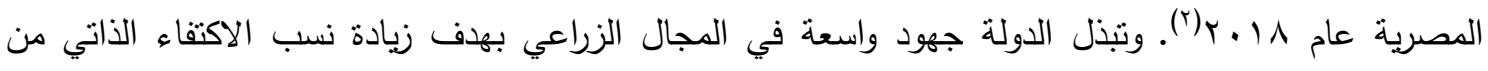

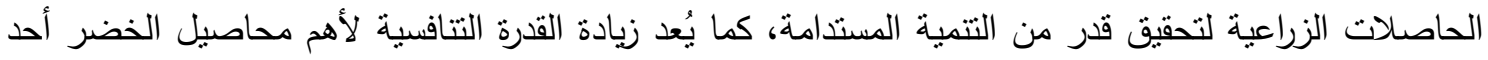

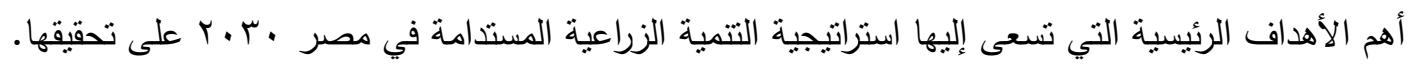
ويُعد الفلفل من محاصيل الخضر الهامة بالعائلة الباذنجانية، ويزرع في مصر في الحقل المكشوف في العروة الصيفية التي يظهر إنتاجها في منتصف مايو ويستمر حتى أوائل نوفمبر ، وفي العروة الخريفية التي تزرع في شهر فير فئري يوليو وأغسطس وسبتمبر ويظهر إنتاجها حتى نهاية ديسمبر • ويندر نواجد الفلفل بالحقل المكشوف اعتبارًا من أوائل يناير حتى منتصف مايو · وييدأ الإنتاج المحمي في تغطية تلك الفترة الحرجة من العام وذلك عن طريق توفير

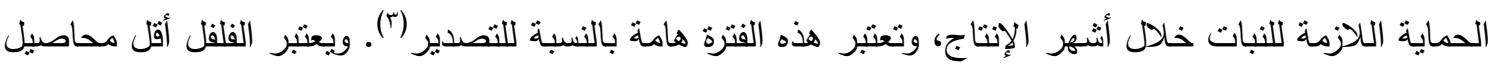
الزراعات المحمية إحتياجًا للعمالة، كذلك يمتاز بسهولة إنتاجه، ولكنه حساس جدًا لدرجة الحرارة الدنيا والعظمى وإن هان إستعادة النباتات لحيويتها ليس بالأمر السهل إذا ما أصييت بصدمات حادة خلال موسم النمو والإنتاج(عأ.

\section{المشكلة البحثية}

على الرغم من اتجاه الدولة في السنوات الأخيرة إلى التوسع في الصادرات الزراعية من محاصيل الخضر إلا أن

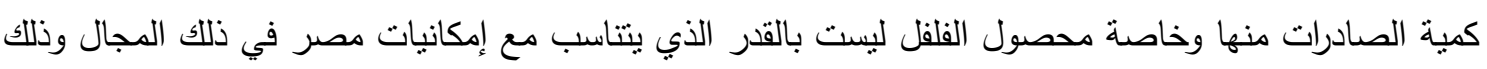

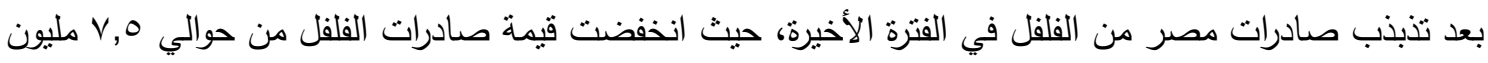

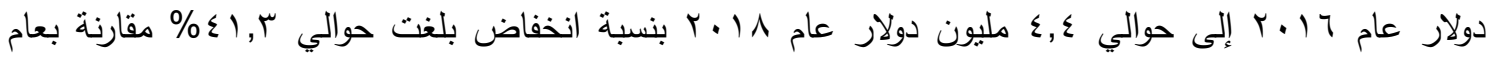

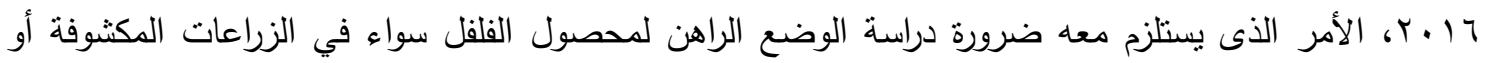


المحمية، والعمل على مضاعفة الإنتاج وفتح أسواق جديدة تتيح لمصر ميزة تتافسية خارجية لتحقيق فائض يساهم في زيادة معدلات من الصادرات الزراعية المصرية وزيادة حصيلة النقد الأجنبى.

\section{الههف من البحث}

يستهدف البحث التعرف على الوضع التتافسي للتجارة الخارجية الزراعية للفلفل المصري في أهم الأسواق العالمية

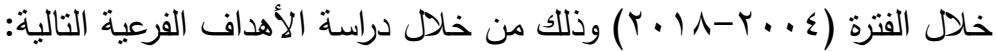

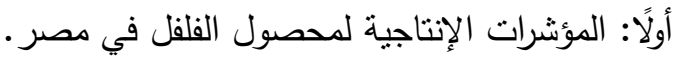

ثانيًا: الكميات المستهلكة ومتوسط نصيب الفرد ونسبة الاكتقاء الذاتي من الفلفل في مصر . ثالثًا: التجارة الخارجية الزراعية ومؤشرات القدرة التتافسية للفلفل.

\section{الأسلوب البحثي ومصادر البيانات}

اعتمد البحث في تحقيق أهدافه على كل من استخدام الأسلوب التحليلي الوصفي والقياسي حيث نم الاعتماد على الاسلوب الوصفي لتحليل الوضع الراهن للفلفل في مصر وفي الأسواق العالمية باستخدام المتوسطات الحسابية، النسب المئوية، مقدار التغير السنوي، ومعدلات النمو السنوي باستخدام معادلات الاتجاه الزمني العام، وكذلك استخدام بعض المؤشرات الاقتصادية المختلفة كمعامل التركز الجغرافي، مؤشرات القدرة التنافسية:

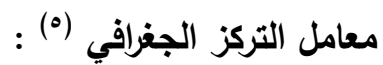
يوضح هذا المعامل مدي تركز التجارة الخارجية لدولة ما لسلعة معينة في سوق واحد أو عدد محدد من الأسواق، مداق ويستخدم معامل جيني "هيرشمان" لحساب درجة التركز الجغرافي للصادرات المصرية من الفلفل خلال فترة زمنية

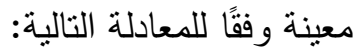

$$
\text { G.C.F. }=\sqrt{\sum_{z=1}^{n}\left[\left(\frac{Y Z}{Y}\right)^{2}\right]} * 100
$$

حيث G.C.F. معامل التزكز الجغرافي (z) : الصادرات من سلعة معينة إلي سوق معينة : Yz Y : إجمالي الصادرات الكلية من السلعة إلي مختلف الأسواق الاسنترادية.

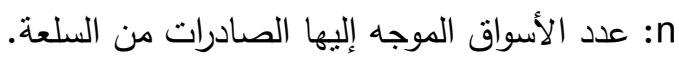

ويصل هذا المعامل إلي أقصي قيمة له وتساوي . . 1 في حالة ما إذا كانت السلعة تصدر إلي سوق واحد فقط من الأسواق الاستيرادية، بينما نقل هذه القيمة كلما نوزعت كمية الصادرات علي عدد كبير من الأسواق الاستيرادية، يعد معامل التركز الجغرافي مرتفعًا إذا كانت قيمته أكبر من • ــ درجة، ويعني ذلك احتمال حدوث نقلبات سعرية شديدة

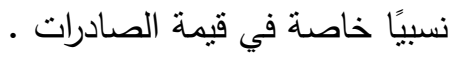
مؤثر النصيب السوقي (؟): يعبر هذا المؤشر عن النسبة بين صادرات الدولة من سلعة ما في أحد أسواقها التصديرية بالقياس إلى الطاقة الإستيعابية الكلية لهذا السوق وُيعد ارتفاع النصيب السوقي لسلعة ما في الأسواق الخارجية إنعكاسًا للقدرة التتافسية

$$
\text { لهذه السلعة ومدى قدرتها على فتح أسواق خارجية. }
$$




$$
M . S_{j k i}=\frac{\mathbf{X j k i}}{\mathbf{X k i}} * 100
$$

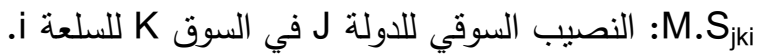

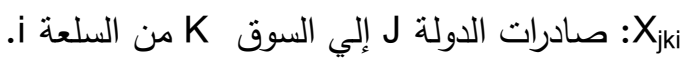
X الواردات الكلية للدولة Ki

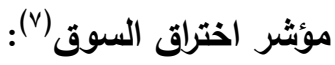

يُعد مؤشر اختراق السوق لسلعة ما من أكثر المقاييس إستخداماً لقياس القدرة التتافسية لأى دولة في تصدير سلعة

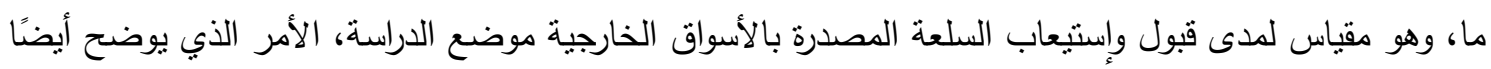

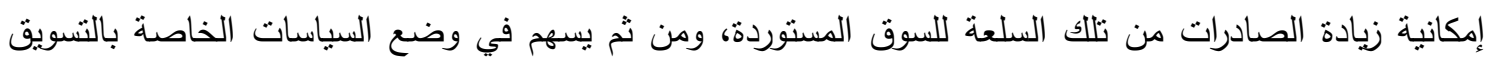
الخارجي للسلعة بكل سوق خارجي، و بمعنى آخر فإن معامل اختراق السوق يدل على وجود سوق خارجي حقيقي للسلعة ومدى قابليته لإسنتعاب كميات إضافية من السلعة، فكلما زادت قيمته دل ذللك على قدرة تلك الك السلعة المنتجة

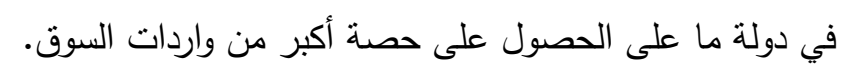

$$
\mathbf{M}_{\mathbf{p}}=\frac{\mathbf{M}_{\mathrm{ijc}}}{\left(\mathbf{Q}_{\mathrm{ij}}+\mathbf{M}_{\mathrm{ij}}\right)-\mathbf{X}_{\mathrm{ij}}} * 100
$$

حيث:Mp : نسبة اختراق السوق (i) من السلعة (j) ).

. (c) : الواردات للسوق (iji

$$
\begin{aligned}
& \text { Qij }
\end{aligned}
$$

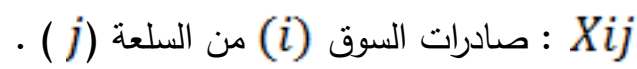

التنافسية السعرية(^) (1) (1)

يعتبر السعر التصديري من المؤشرات الهامة لجذب المزيد من الأسواق التصديرية في ظل التقارب النوعي للسلعة

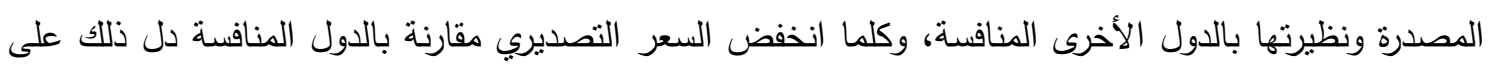
وجود ميزة سعرية وقدرة تتافسية أفضل، والسعر التصديري لا يعكس فقط التكاليف الإنتاجية للسلعة ولكنه يتأثر بعوامل أخرى مثل تكاليف النقل والضرائب، موعد التصدير ، وغيرها من التكاليف المرتبطة بعملية التصدير .

$$
\mathbf{P}_{\mathbf{A j}}=\frac{\mathbf{P}_{\mathbf{i}}}{\mathbf{P}_{\mathbf{c}}} * 100
$$

حيث PAj : النسبة بين سعر الدولة المصدرة والدولة المنافسة.

$$
\text { Pع : Pr }
$$$$
\text { : Pc }
$$

واعتمد البحث في تحقيق أهدافه على البيانات الثانوية التي تم تجميعها من النشرات والدوريات الصـادرة عن الإدارة

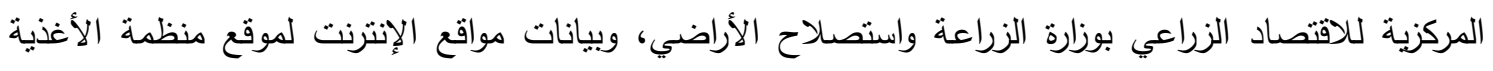


والزراعة العالمية، موقع التجارة الدولية للأمم المتحدة، بالإضافة إلى البحوث التي لها علاقة بموضوع البحث.

\section{النتائج البحثية ومناقثتها}

أولاً: المؤثشرات الإنتاجية لمحصول الفلفل في مصر:

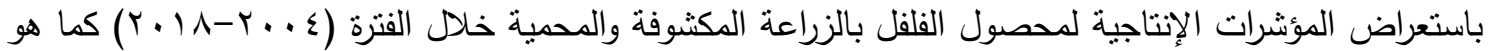

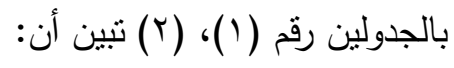

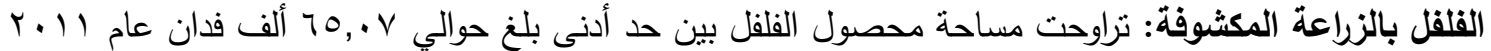

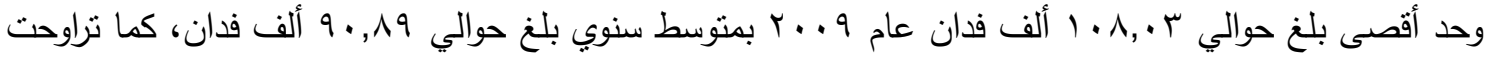

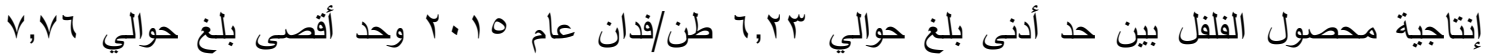

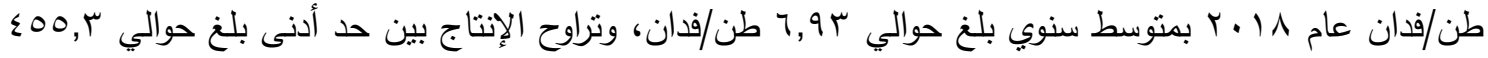

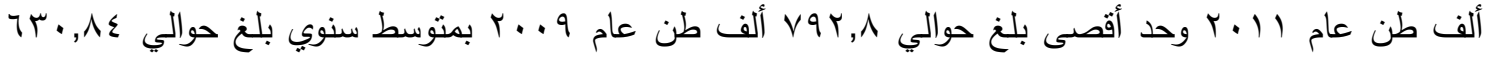
ألف طن. وبتقدير معادلة الاتجاه الزمني العام تبين عدم ثبوت المعنوية الاحصائية لمساحة وإنتاجية وإنتاج

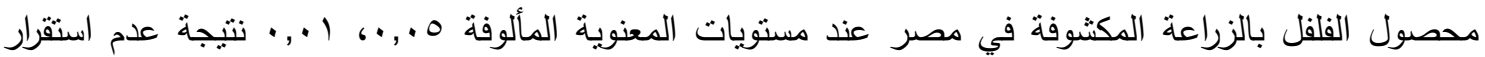
البيانات الخاصة بالمؤشرات الإنتاجية السابقة خلال فترة الدراسة.

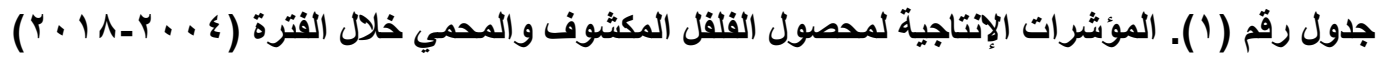

\begin{tabular}{|c|c|c|c|c|c|c|c|c|}
\hline \multicolumn{2}{|c|}{ الإجمالي } & \multicolumn{3}{|c|}{ القلقل بالأراضي المحمية } & \multicolumn{3}{|c|}{ القلقل بالأراضي المكشوفة } & \multirow[b]{2}{*}{ السنوات } \\
\hline ألف طن الإنتاج & فدان & الف طن الإنتاج & طن/إذاجنة & المسـاحة فدان & الف طن الإنتاج & طن/إناجية & فدان & \\
\hline$\varepsilon \wedge 1$, & $\vee 1.9 \wedge, 7$ & $1 \pi, 7$ & M & $\varepsilon r \wedge, T$ & $\varepsilon T V, \varepsilon r$ & 7,71 & V.77. & $r \cdot \varepsilon$ \\
\hline $07 ., \varepsilon$ & $\wedge \cdot \wedge r q, r$. & $1 v, 1$ & $r V, r$ & $\leq 09, r$ & $0 \leqslant \mu, \mu \leqslant$ & $7, \vee 7$ & A.rV. & $r . .0$ \\
\hline$V \cdot 7, \wedge$ & ..人rl, & $r, r$ & $\varepsilon 1$ & $0 \leq 1$ & $\neg \wedge \varepsilon, 7 \leq$ & $7, \lambda$ & I. r r . & $r \ldots r$ \\
\hline $7 \wedge 1$, & $90 \wedge r \varepsilon, 1$ & $r q, r$ & $\leqslant 1,0$ & $V \cdot \varepsilon, 1$ & 701, Ar & $7, \wedge 0$ & $901 \mathrm{r}$. & $r . . v$ \\
\hline$v \leqslant q$, & r. & $\leqslant 0,7$ & $\varepsilon 1, r$ & $11 \cdot 7,1$ & $V \cdot r, \varepsilon)$ & $7,9 V$ & 1.997. & $r . \wedge$ \\
\hline Nro,r & $1 \cdot \wedge \wedge \wedge \vee, 1$ & O & $r v, q$ & Nov, 1 & $\vee q Y, \wedge \varepsilon$ & V & I.A.r. & $r \ldots q$ \\
\hline $79 \cdot, \varepsilon$ & 9 qroq, 7 & $r \varepsilon, 7$ & r, & 909,7 & $700, \wedge \varepsilon$ & $\vee, 1 \wedge$ & $91 \leq \ldots$ & r.l. \\
\hline$\leqslant 91, V$ & $77) \wedge 7,1$ & r & r & 1117,1 & $\varepsilon 00, \mu r$ & $\mathrm{~V}, \cdot$ & $70 . V$. & $r .11$ \\
\hline הTr, & qVYAl, & r & r, & $r \leq V i$ & $70 ., 00$ & $7, \wedge\rceil$ & $9 \leq \wedge 1$. & $r .1 T$ \\
\hline$T r V, \cdot$ & NOr $\leqslant V, V$ & 71,7 & $\leqslant 7, \varepsilon$ & ITrv, & $070, \leqslant r$ & $7, V T$ & $\Lambda \varepsilon \cdot r$. & $r .1 T$ \\
\hline $7 \vee q, Y$ & $q \curlyvee 0 \wedge q, q$ & $V \vee, q$ & LT, & $1 \vee 99,9$ & $7.1, r_{q}$ & $7,7 r$ & $9 . \vee 9$. & $r .1 \leq$ \\
\hline$T \wedge \wedge, r$ & $9 \wedge 99 \leq, q$ & vr, $q$ & $\varepsilon 1,7$ & $1 \vee \vee \varepsilon, q$ & $T) \varepsilon, Y V$ & r & QYYT. & $r .10$ \\
\hline VYA, 1 & $q \wedge \mu \mid q, \wedge$ & $\mathrm{VT}, \wedge$ & $r q, 1$ & $1 \wedge \wedge 9, \wedge$ & $T 0 \leqslant, Y T$ & $7, \vee \wedge$ & ૧૫ะน. & $r .17$ \\
\hline$v \leqslant r, q$ & $9 r q r o, 1$ & 77,0 & rv, & IVAo, 1 & $T \vee T, \leqslant Y$ & $v, r v$ & $9) \wedge \varepsilon$. & $r .1 \mathrm{~V}$ \\
\hline$\wedge) \wedge, r$ & $q \wedge \mid r \vee, 0$ & $V Y, 0$ & $r q, 0$ & INHV,o & $V \leqslant 0, V$ & $V, V T$ & q7т.. & $r .11$ \\
\hline $71 \cdot, Y_{1}$ & 94101,0 & $\varepsilon q, \mu V$ & $r \wedge, 7 r$ & $|r v|, r$. & $\pi \cdot, \Lambda \varepsilon$ & 7,91 & 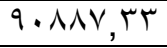 & المتوسط \\
\hline
\end{tabular}

المصدر: جُمعت وحسبت من: وزارة الزراعة، قطاع الثئون الاقتصادية، الإدارة المركزية للاقتصاد الزراعى، نشرة الاحصاءات الزراعية،

القاهرة، أعداد متفرقة. 
جدول رقم (Y). معادلات الاتجاه الزمنى العام لمساحة و إنتاج وإنتاجية القلقل في مصر خلال الفترة (؟ . . ؟ـ (r.1)

\begin{tabular}{|c|c|c|c|c|c|}
\hline معدل النمو\% & ف & 广ر & المعادلة & الوحدة & المتغير التابع \\
\hline م.غ & (亡. & $\cdot, \cdot 7$ & 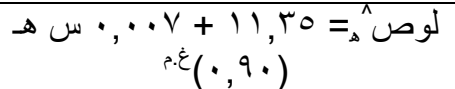 & المساحة & \multirow{3}{*}{ 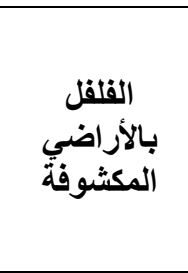 } \\
\hline م.غ & ( & $\cdot, \wedge$ & 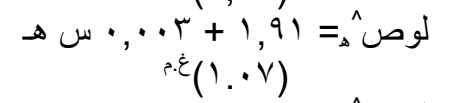 & الإنتاجية & \\
\hline غ غ & r & $\cdot, \cdot 9$ & لوص & الإنتاج & \\
\hline $11, r$ & ${ }^{* *} 00,94$ & $\cdot, \wedge)$ & 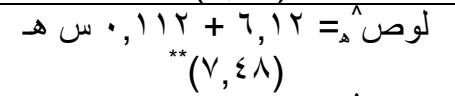 & المساحة & \multirow{3}{*}{ القلقل المحمي } \\
\hline م.غ & (1) 17 & $\cdot, \wedge$ & 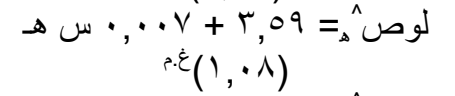 & الإنتاجية & \\
\hline 11,9 & **or, or & $\cdot, \wedge)$ & 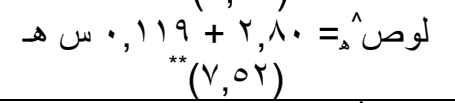 & الإنتاج & \\
\hline غ.غ.م. & ri & $\cdot, \cdot 1$ & 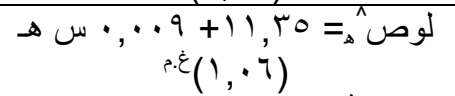 & المساحة & \multirow{2}{*}{ إجمالي القلقل } \\
\hline غ. & & $\cdot, r T$ & 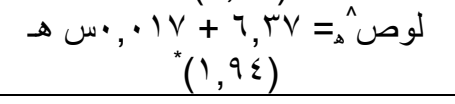 & الإنتاج & \\
\hline
\end{tabular}

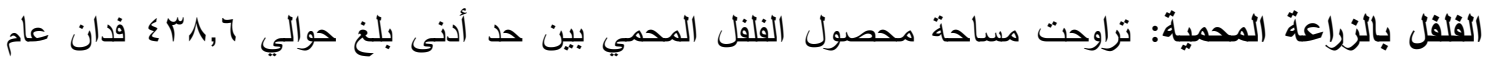

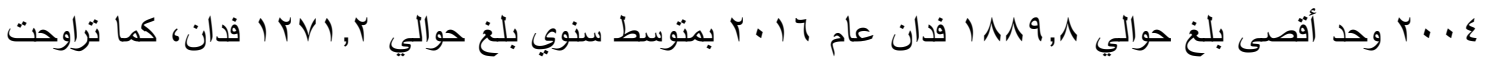

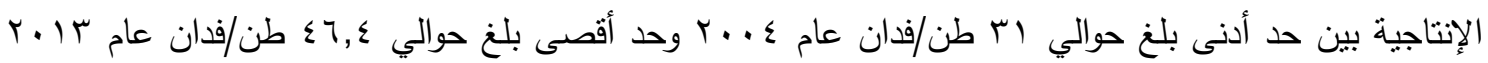

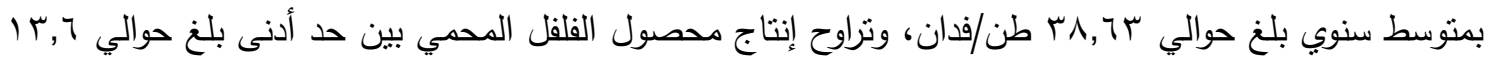

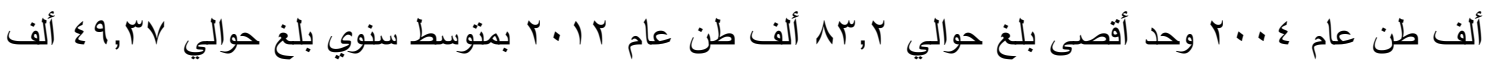

وبتقدير معادلة الاتجاه الزمنى العام تبين أن مساحة وإنتاج محصول الفلفل المحمي نأخذا اتجاها عامًا متزايدًا

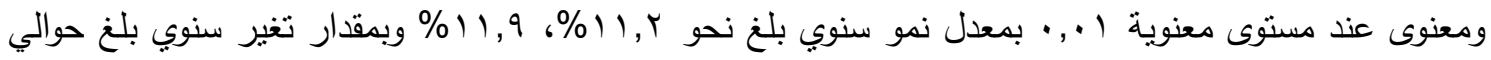

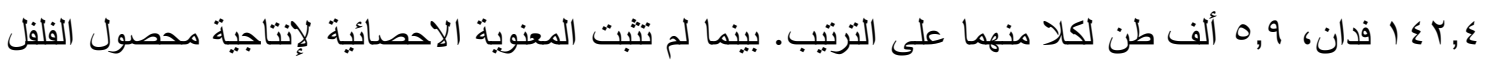

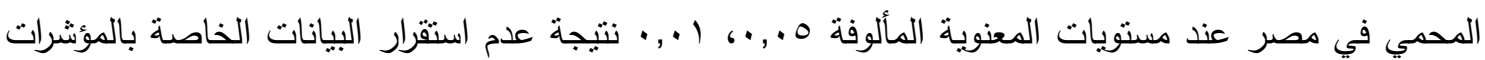
الإنتاجية السابقة خلال فترة الدراسة. إجمالي الفلقل في مصر : تراوح إجمالي مساحة محصول الفلفل في مصر بين حد أدنى بلغ حوالي r. ب7 ألف فدان

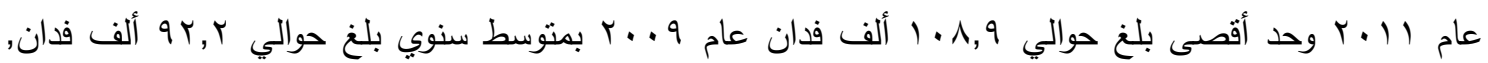

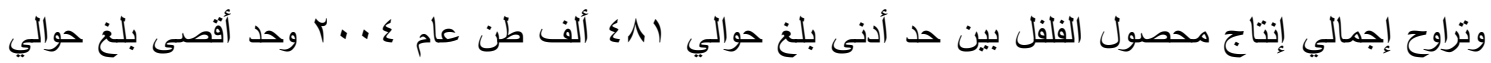

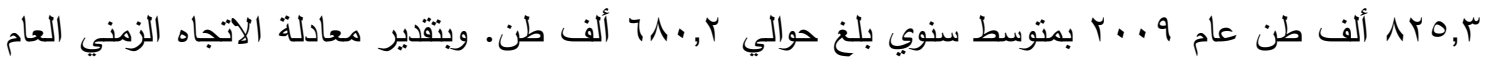

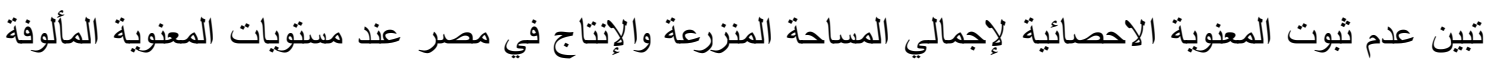
ه ., ..، ا .., • نتيجة عدم استقرار البيانات الخاصة بالمؤشرات الإنتاجية السابقة خلال فترة الدراسة. 
ثانياً: الكميات المستهكة ومتوسط نصيب الفرد ونسبة الاكتفاء الذاتى من الفقل في مصر : تبين من استهلاك محصول الفلفل في مصر كما هو موضح بالجدول رقم (r) أنه نراوح بين حد أدنى بلغ حوالي

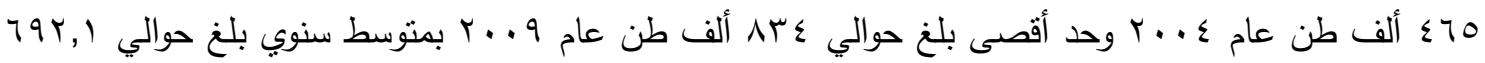

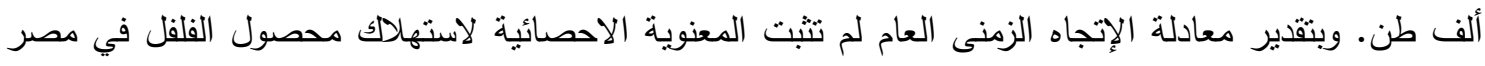
عند مستونات المعنوية المألوفة. كما إتضح أن منتسط نصيب الفرد من الفلفل نراوح بين حد أدنى بلغ حوالي

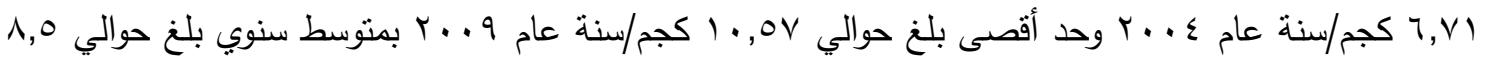
كجم/سنة، وبتقدير معادلة الإتجاه الزمنى العام تنين عدم تتبوت المعنوية الاحصائية لمتوسط نصيب الفرد من بن

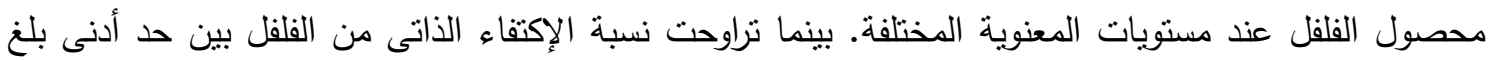

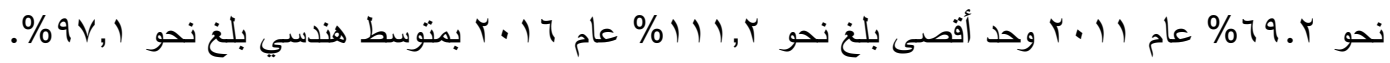
ثالثاً: التجارة الخارجية الزراعية ومؤشرات القدرة التنافسية للقلقل المصري :

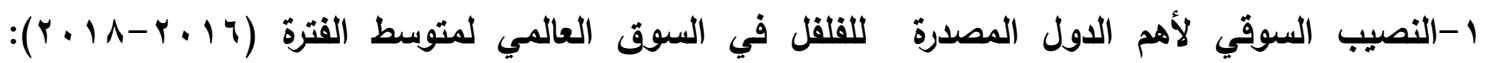

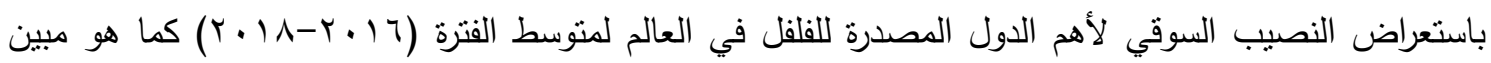
بالجدول رقم (ع) نتين أن النصيب السوقي للمكسيك في السوق العالمي يأتى في المرتبة الأولى حيث بلغت كمية

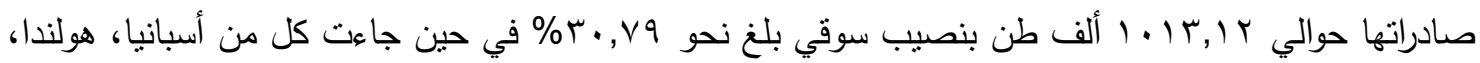

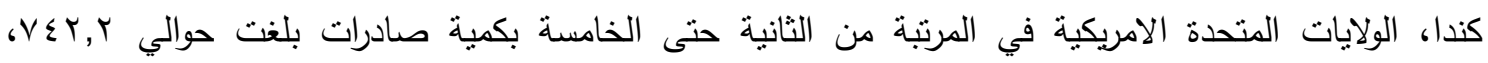
○, . إجمالي واردات العالم لكل منهم على الترتيب. وقد جاءت تركيا، الصين، المغرب، إسرائيل، الأردن

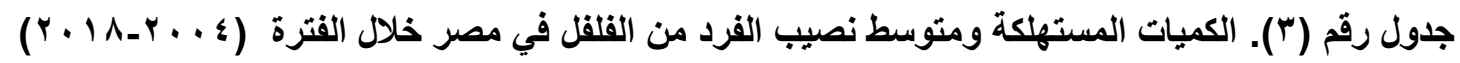

\begin{tabular}{|c|c|c|c|}
\hline نسبة الإكتفاء الأتى \%\% & متوسط نصيب الفرد كجم/ سنة & الكمية المستهلكة ألف طن & السنوات \\
\hline $1 \cdot r, \varepsilon$ & $7, \vee 1$ & $\leqslant 70$ & $r \ldots \varepsilon$ \\
\hline$\wedge q, r$ & $v, 79$ & $0 \leqslant \pi$ & $r \ldots o$ \\
\hline 99,1 & $9,9$. & VIT & $r \ldots r$ \\
\hline$q \vee, \wedge$ & $q, \leqslant 0$ & 797 & $r \ldots v$ \\
\hline 99,1 & $1 \cdot, 0$ & Vor & $r \ldots \wedge$ \\
\hline $99, \cdot$ & $1 ., 0 \mathrm{~V}$ & ᄉr & $r \ldots q$ \\
\hline $99, r$ & $\wedge, \wedge \varepsilon$ & 797 & $r+1$. \\
\hline $79, r$ & $\Lambda, \wedge \mu$ & vil & $r+11$ \\
\hline $99, r$ & 1,97 & $V \varepsilon$. & $r+1 r$ \\
\hline $9 \wedge, 9$ & $\vee, \varepsilon q$ & צז & $r+1 r$ \\
\hline $1 \cdot 1,1$ & $v, v \leqslant$ & TVY & $r+1 \varepsilon$ \\
\hline $9 \wedge, r$ & $\vee, \wedge \wedge$ & $v \cdot 1$ & $r+10$ \\
\hline $111, r$ & $\vee, r$. & 700 & $r+19$ \\
\hline 91,0 & $\vee, 9 Y$ & Vos & $r+I V$ \\
\hline $1 \cdots, 9$ & $\Lambda, r \leqslant$ & 111 & $r+11$ \\
\hline $9 \vee, 7$ & $\Lambda, 0$. & 794,1 & المتوسط \\
\hline
\end{tabular}

"منوسط هندسي. المصدر: ا- وزارة الزراعة. قطاع الثئون الاقتصادية، الإدارة المركزية للاقتصاد الزراعى، نثرة الميزان الذذائ،

القاهرة، أعداد متفرقة. ץ- الجهاز المركزى للتعبئة العامة والاحصاء، النشرة السنوية للإنتاج وحركة التجارة الخارجية والمتاح للاستهلاك من السلع الذذائية، 


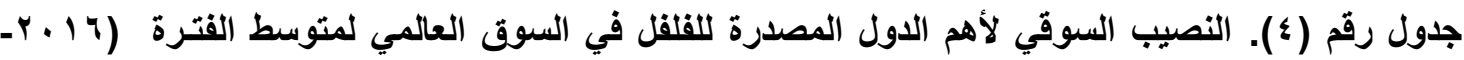
(r+l)

\begin{tabular}{|c|c|c|c|}
\hline الترتيب & النصيب السوقي \% & كمية الصادرات (ألف طن) & 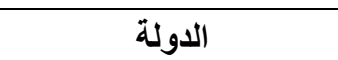 \\
\hline الأول & $r \cdot, v_{q}$ & $1.1 r, 1 r$ & المكسيك \\
\hline الثانى & $r r, 00$ & $V \leqslant r, r$. & أسبانيا \\
\hline الثالث & $1 \pi, 70$ & $\leqslant \leqslant 9,0$ & هولندا \\
\hline الر ابع & $\varepsilon, \varepsilon \uparrow$ & $1 \leq 7, \wedge r$ & كندا \\
\hline الخامس & $r, 7 r$ & $M \Lambda, v$. & الولايات المتحدة الأمريكية \\
\hline السادس & $r, r \varepsilon$ & $1.7,01$ & تركيا \\
\hline السابع & $r, q$. & 90,0 r & الصين \\
\hline الثامن & $r, r \Lambda$ & vo, 10 & المغرب \\
\hline التاسع & $r, 1 r$ & $79,9$. & إسرائيل \\
\hline العانشر & $1,0 \varepsilon$ & $0 ., 07$ & الأردن \\
\hline الو احد و الثلاثثن & $\cdot, r V$ & $\wedge, \vee_{0}$ & مصر \\
\hline & $1 \ldots$ & $r Y q \cdot, V \leq$ & إجمالي واردات العالم \\
\hline
\end{tabular}

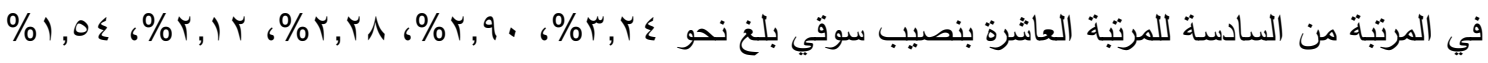
على الترتيب، وبلغت إجمالي صادرات هذه الدول العشر حوالي r,AV مليون طن بنصيب سوقي بلغ نحو

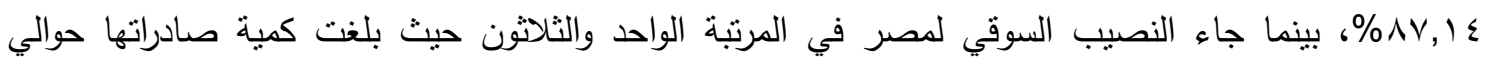
^, Vo

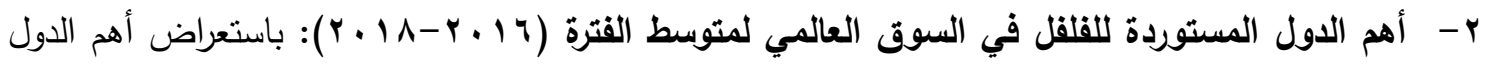

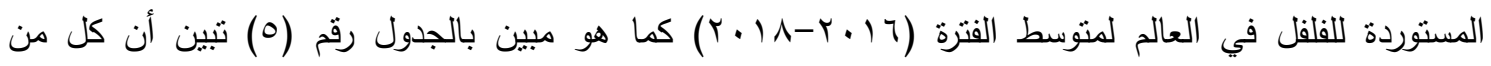

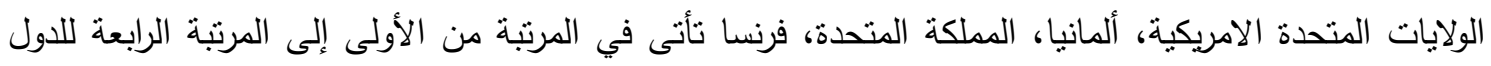

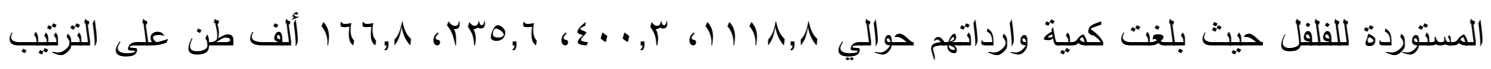

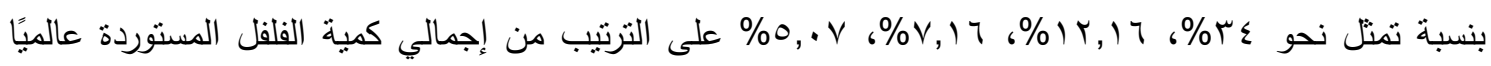

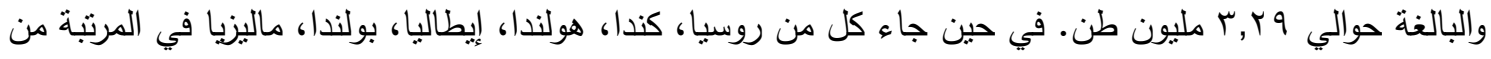

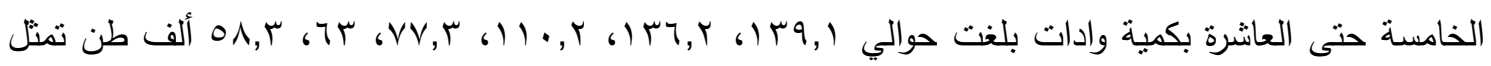

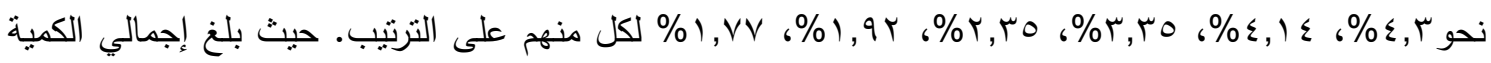

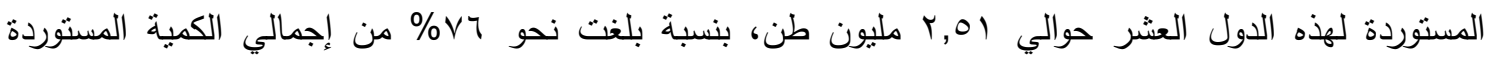
عالميًا. 


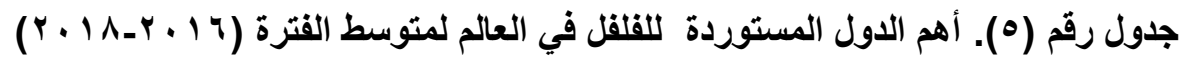

\begin{tabular}{|c|c|c|}
\hline$\%$ & الكمية (ألف طن) & الدولة ل \\
\hline$\Gamma \varepsilon_{.}$. & $111 \wedge . \vee \wedge$ & الولايات المتحدة الأمريكية \\
\hline 14.17 & $\varepsilon \cdots . r$ & ألمانيا \\
\hline 8.17 & rTO.TY & المملكة المتحدة \\
\hline $0 . V$ & 174.81 & فرنسا \\
\hline$\varepsilon . r T$ & 149.11 & روسيا \\
\hline$\varepsilon .1 \varepsilon$ & (T., & كندا \\
\hline ס. T. & $11 \cdot .10$ & هولندا \\
\hline o tro & $V Y . Y \wedge$ & إيطاليا \\
\hline $1.9 r$ & 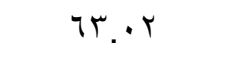 & بولندا \\
\hline I.VV & $0 \wedge . Y T$ & ماليزيا \\
\hline VI. I $\varepsilon$ & Y0.0. $\varepsilon \Lambda$ & المجموع \\
\hline rT.AT & VAO.YT & باقي الدول \\
\hline $1 \ldots$ & rYq..V & إجمالي واردات العالم \\
\hline
\end{tabular}

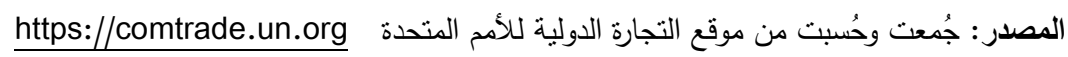

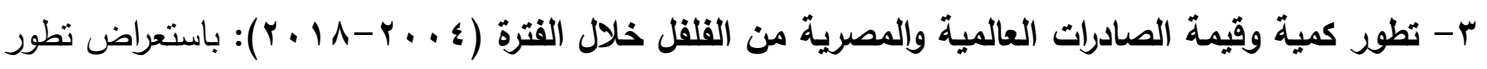

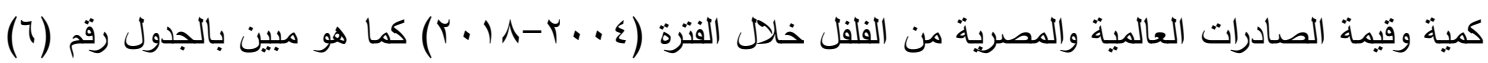

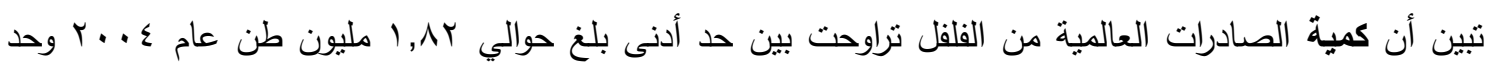

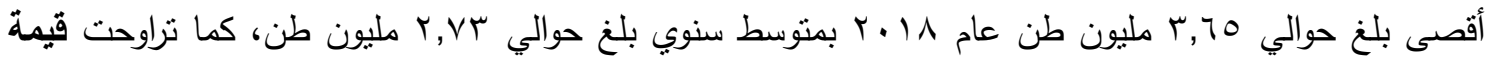

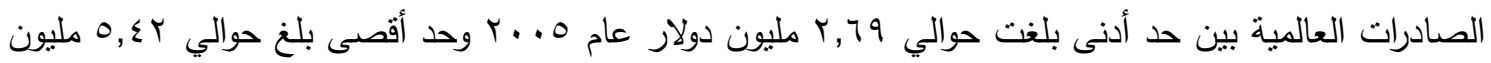

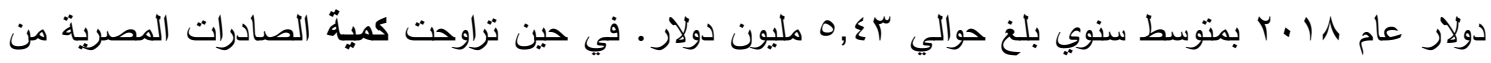

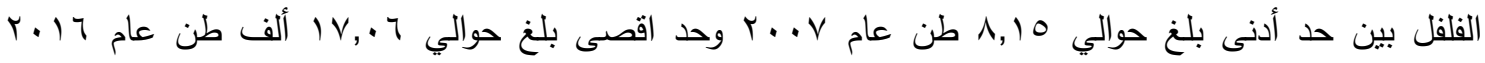

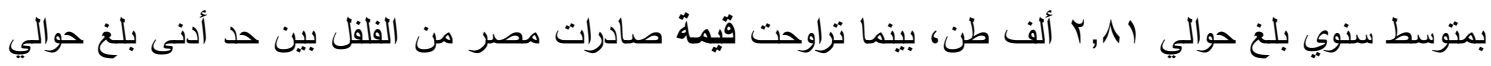

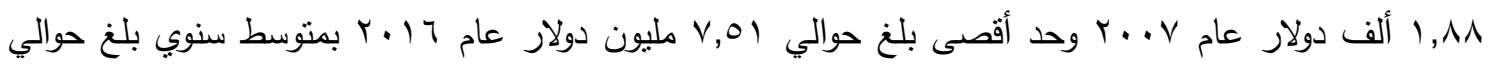

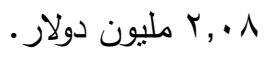

وبتقدير معادلات الإتجاه الزمنى العام لكمية وقيمة الصادرات العالمية من الفلفل كما هو مبين بالجدول رقم (v)

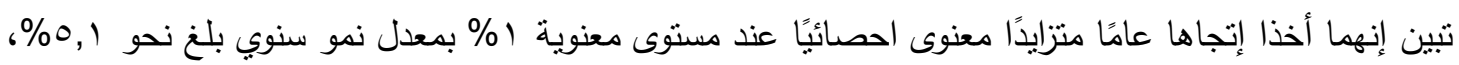

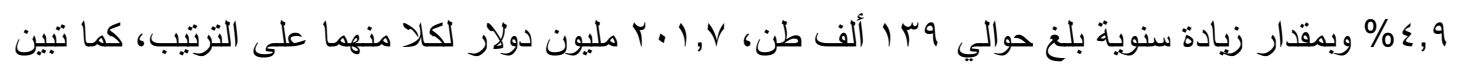

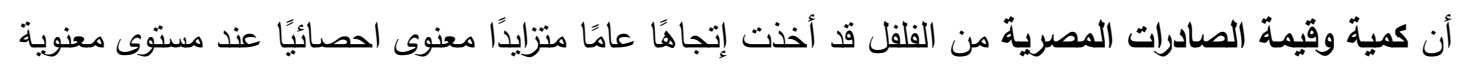

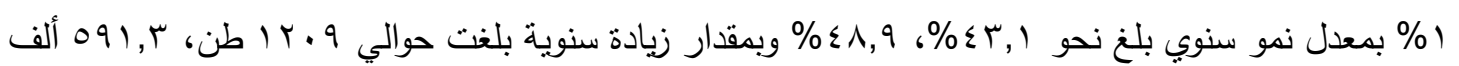
دولار لكلا منهما على الترتيب. 
جدول رقم (7). تطور كمية وقيمة الصادرات الزراعية العالمية والمصرية من الفلقل خــلال الفتـــرة $(r+1 \Lambda-r+\varepsilon)$

\begin{tabular}{|c|c|c|c|c|}
\hline \multicolumn{2}{|c|}{ الصادرات المصرية } & \multicolumn{2}{|c|}{ الصادرات العالمية } & \multirow[b]{2}{*}{ السنوات } \\
\hline قيمة & كمية & قيمة & كمية & \\
\hline ألف دولاز & طن & ألف دولار & طن & \\
\hline $07,{ }_{0}$ & $9 V, Y \varepsilon$ & YVTTVOT & IAYY. EA & $r \ldots \varepsilon$ \\
\hline $0, \wedge$. & $10, Y \varepsilon$ & 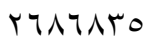 & INTYTVA & $r \ldots$ \\
\hline 11,00 & $r \cdot, 1 V$ & 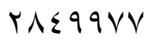 & 190VATA & 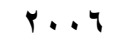 \\
\hline $1, \wedge \wedge$ & 1,10 & $r \leqslant \varepsilon r \neg \wedge q$ & YITT人YE & $r \ldots v$ \\
\hline$v \leq 1,91$ & $1 V \cdot q, r_{0}$ & $\varepsilon . \varepsilon .070$ & rrvoNi. & $r \ldots \Lambda$ \\
\hline 1047,91 & $117 \cdot, 7$ & & Y0YAI7. & $r . . q$ \\
\hline $100 \leqslant, Y T$ & 1.7, & 5.04 .99 & rON.MIT & $r+1$. \\
\hline$q \wedge 7, \cdot r$ & ס ז,.., & ET019人r & 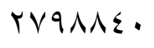 & 4.11 \\
\hline ع & $011, \cdot V$ & $\varepsilon r .9 .91$ & rAY9OHA & $r \cdot 1 r$ \\
\hline $99 \vee, 9 \leq$ & سד, & $\Sigma \wedge r \leqslant \wedge \leqslant r$ & rq $\varepsilon .0 . \varepsilon$ & $r .1 r$ \\
\hline$\leqslant 7 \cdot \wedge, \leqslant 7$ & r Y $\varepsilon, \lambda r$ & $\leqslant \vee q r \wedge .1$ & $\Gamma|V \cdot \lambda| \varepsilon$ & $r \cdot 1 \varepsilon$ \\
\hline YqV।,人т & $7 r \leq q, \wedge \wedge$ & $\varepsilon O \wedge \vee \wedge O \vee$ & 9צ' & $r .10$ \\
\hline $10.7,71$ & IV & $\Sigma 9 \vee \wedge 99 Y$ & & $r .17$ \\
\hline $0 \ldots 7, V_{7}$ & $\varepsilon .0 \vee, 9 \Lambda$ & 0.11040 & ror人tтV & $r .1 \mathrm{~V}$ \\
\hline$\varepsilon \varepsilon r \cdot, V Y$ & צ० & O\&YONVT & rฯะVYq. & $r \cdot 11$ \\
\hline$r \cdot \Lambda I, Y r$ & $r \wedge \cdot 0, r \varepsilon$ & $\varepsilon 110 \vee \wedge \varepsilon$ & YVYOQE. & المتوسط \\
\hline
\end{tabular}

جدول رقم (V). معادلات الإتجاه العام الزمنى لكمية وقيمة الصادرات العالمية والمصرية من الفلقل خلال الفترة $(r+1 \Lambda-r+. \varepsilon)$

\begin{tabular}{|c|c|c|c|c|}
\hline معدل النمو\%\% & ف & J & المعادلة & المتغير التابع \\
\hline 0,1 & * * * & $\cdot, 91$ & 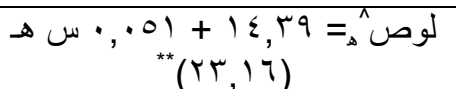 & كمية الصادرات العالمية \\
\hline$\varepsilon, 9$ & ${ }^{* *}, 1 r, 7$ & $\cdot, 9$. & لوص ^ = & قيمة الصادرات العالمية \\
\hline$\varepsilon r, 1$ & $* * Y \vee, \Sigma)$ & $\cdot, 7 \Lambda$ & 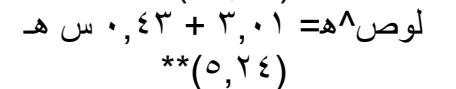 & كمية الصادرات المصرية \\
\hline$\varepsilon \wedge, q$ & $* * Y \curlyvee, \wedge \varepsilon$ & $\cdot$, TV & لوص له & قيمة الصادرات المصرية \\
\hline
\end{tabular}

" معنوى عند ا.,. المصدر: جُمعت وحُسبت من جدول رقم (؟).

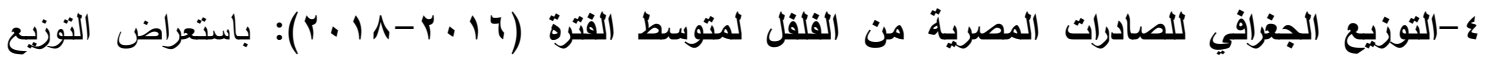
الجغرافي للصادرات المصرية من الفلفل كما هو مبين بالجدول رقم (^) تبين أن أهم الدول المستوردة للفلفل المصري تتمنل في السعودية، المجر، روسيا، ليبيا، الكويت تأتى هذه الدول من المرتبة الأولى إلى المرتبة الخامسة

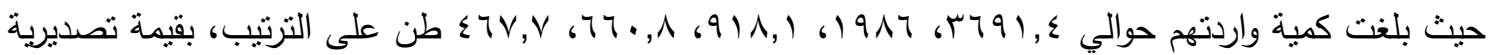

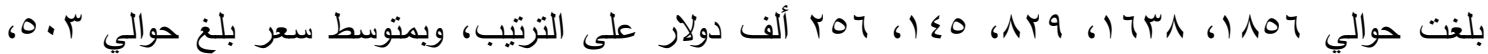

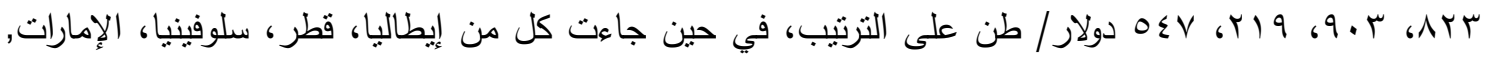

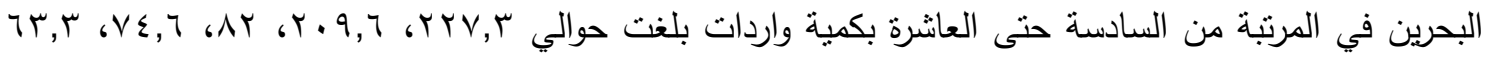

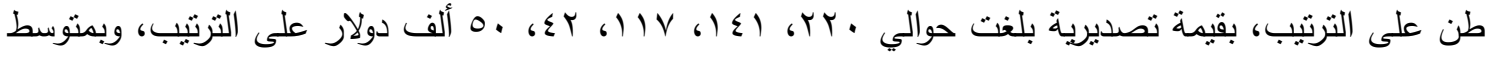


سعر بلغ حوالي 974، 7V0، Y

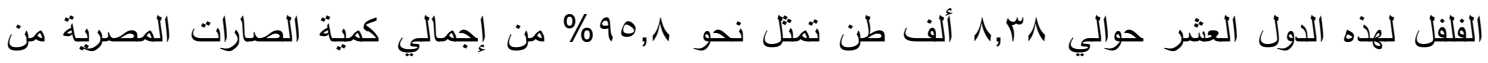

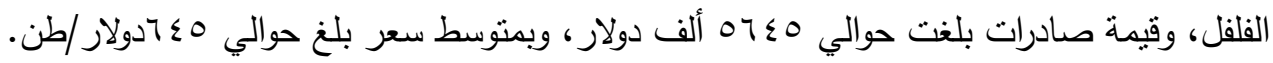

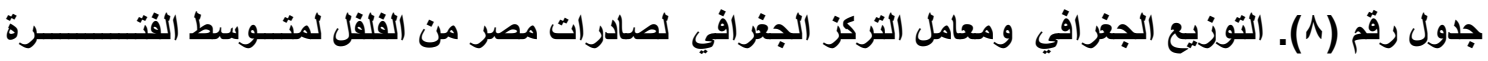
$(r \cdot 1 \Lambda-r \cdot 17)$

\begin{tabular}{|c|c|c|c|c|}
\hline سعر التصدير (فوب) & قائفة الصادرات & معامل التركز الجغرافي & (طن) & الدولة \\
\hline $0 . Y, Y$ & 1100,1 & 09,71 & r7q1, & السعوية \\
\hline$\Delta r \leqslant, q$ & $17 r \Lambda, r$ & $r q, \cdot 1$ & 1917,. & المجر \\
\hline $9 \cdot r, 0$ & $\wedge \nmid \wedge, \uparrow$ & ro,qr & 911,1 & روسيا \\
\hline Y\, ^ & $1 \leq \varepsilon, 7$ & $r r, Y)$ & $74 \cdot, \wedge$ & ليبيا \\
\hline $0 \leqslant 7,0$ & $r 00, T$ & $1, \vee_{0}$ & $\leq T V, V$ & الكويت \\
\hline 970,1 & r) 9,7 & $\checkmark, r r$ & rYV,r & ايطاليا \\
\hline$T V \leqslant, 0$ & $1 \leqslant 1, \varepsilon$ & $r, \cdot \Lambda$ & $r \cdot 9,7$ & قطر \\
\hline $1 \leq r r, r$ & $117, \mathrm{~V}$ & $r, Y V$ & $\Delta r$, & سلوفينيا \\
\hline $07 \varepsilon, 1$ & $\varepsilon r, 1$ & $1, r)$ & $V \varepsilon, 7$ & الإمارات \\
\hline V91, 9 & $0 \cdot, r$ & 1,79 & זr, & البحرين \\
\hline $9 \varepsilon, 0$ & ror, 1 & $0, \leqslant 9$ & r79), ₹ & دول أخرى \\
\hline$T \leq 0, r$ & $0 T \leqslant \leqslant, V$ & - & $\Lambda V \leqslant V, 0$ & إجمالي صادرات مصر \\
\hline
\end{tabular}

جاء معامل التركز الجغرافي لصادرات مصر من الفلفل المصري إلي السعودية في المرنبة الأولى حيث بلغ نحو

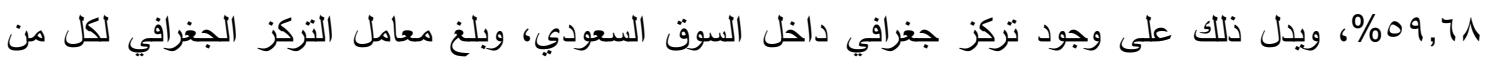

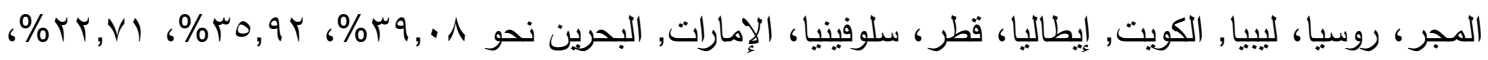

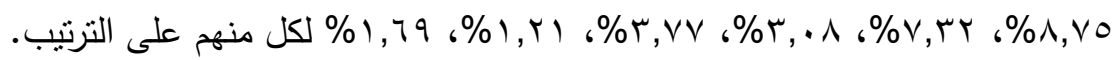
ويتضح مما سبق أن أهم الدول المستوردة للفلفل في السوق العالمي مثل الولايات المتحدة وألمانيا والمملكة المتحدة وفرنسا وكندا لم تكن من أهم الدول المستوردة للفلفل المصري، ومن ثم لابد من الإهتمام بهذه الأسواق والعمل على فئ ولى فتح أسواق جديدة وتتمية صادرات مصر من الفلفل إليها.

ه- مؤثرات القدرة التنافسية لصادرات مصر من الفلقل: باستعراض كمية الإنتاج والصادرات والواردات العالمية

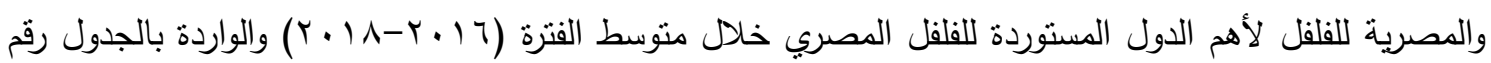
(9) تبين أن كمية الإنتاج المحلي لأهم الدول المستوردة للفلفل المصري والتي تتمنل في المجر ، ليبيا، الكويت،

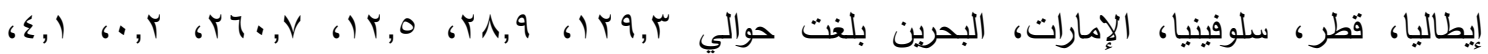
1,،,_ألف طن على الترتيب، في حين بلغت كمية صادرات الفلفل لكل من السعودية، المجر، روسيا،

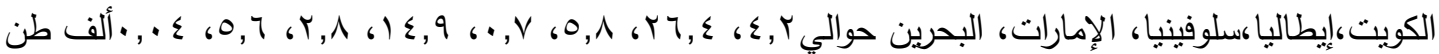




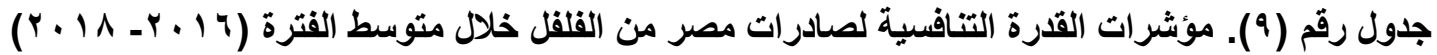

\begin{tabular}{|c|c|c|c|c|c|c|}
\hline \multirow{2}{*}{ السوق اقتراق } & \multirow{2}{*}{ السوقي النصيب } & \multicolumn{2}{|c|}{ كمية الواردات (طن) } & \multirow{2}{*}{ كمية صادرات الدولة (طن) } & \multirow{2}{*}{ كمية الإنتاج } & \multirow{2}{*}{ 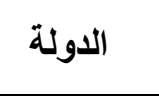 } \\
\hline & & من مصر & من العالم & & & \\
\hline $9,9 V$ & $1 Y, 07$ & Y010, M & rqr^q,0 & $\varepsilon I V \cdot, 0$ & $\cdot$, & السعودية \\
\hline$\cdot, 0 \leqslant$ & IT, & $7 \leq \cdot, 99$ & $101 r 0, r$ & rTrOV, & 1 ห9rา9 & المجر \\
\hline$\cdot, \mathrm{V} \leq$ & $\cdot, 77$ & $9 \wedge r, \vee \leqslant$ & $\mid r q 411,1$ & $O \wedge Y V, 0$ & $\cdot, \cdots$ & 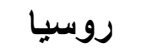 \\
\hline${ }^{*}-$ & $77, \cdot r$ & ${ }^{*}-$ & $\cdots, V$ & $\cdot$, & YA90V & ليييا \\
\hline $1, \cdot \varepsilon$ & $r, \varepsilon$. & rYq, I & $1901 r, r$ & $79 r, r$ & KO.r & الكويت \\
\hline$\cdot, r$ & $\cdot, r q$ & $11 \cdot .09$ & $\mathrm{VPOT}, \mathrm{V}$ & $1 \leq 947, \varepsilon$ & 47.791 & ايطاليا \\
\hline 1,99 & $1, \leqslant r$ & r97, 0. & $1 \leq 71 \vee, 1$ & •, & $r \leqslant 9$ & قطر \\
\hline$\cdot, 1 \mathrm{~V}$ & $1, \cdot V$ & 10,09 & $\vee 709,1$ & rAlr, & EIYฯ & 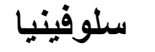 \\
\hline$\cdot, \wedge)$ & $\cdot, 17$ & rNI, r. & EV०T,$r$ & $07 \cdot 7, \mathrm{~V}$ & $0.0 \mathrm{~V}$ & 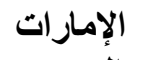 \\
\hline \multirow[t]{6}{*}{$r, 11$} & $\cdot, \lambda r$ & $r \leq 1,1 \leq$ & $\vee \vee \vee q, r$ & $r \Lambda, 0$ & 1.0 & البحرين \\
\hline & & & & \multicolumn{3}{|c|}{ *لم تتوافر بيانات ليييا لحساب مؤشر اختراق السوق. } \\
\hline & & & & \multicolumn{3}{|c|}{ 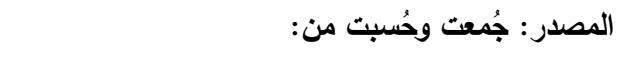 } \\
\hline & & & & & \multicolumn{2}{|c|}{ (1) البيانات الواردة بالجدول رقم ^.. } \\
\hline & & & & \multicolumn{3}{|c|}{ 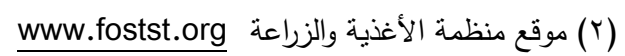 } \\
\hline & & & https://c & rade.un.org & 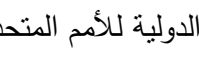 & 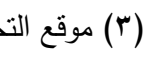 \\
\hline
\end{tabular}

على الترتيب، وبلغ إجمالي كمية الواردات العالمية من الفلفل لكل من السعودية، المجر، روسيا، ليبيا، الكويت،

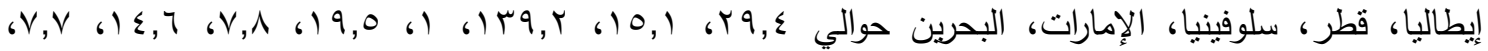

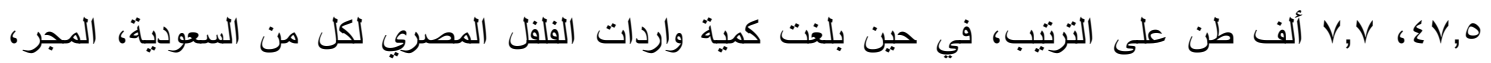

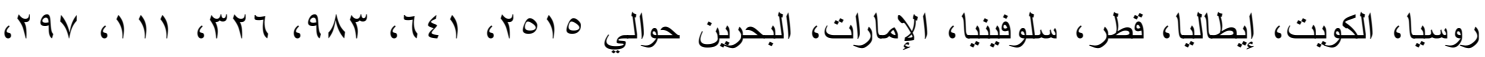

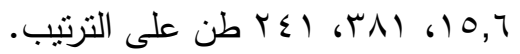
ويتقدير مؤثرات القدرة التنافسية تبين مايلي: لترني - النصيب السوقي لصادرات القلقل المصري في أهم الأسواق العالمية: يتضح أن أكبر متوسط نصيب سوقي

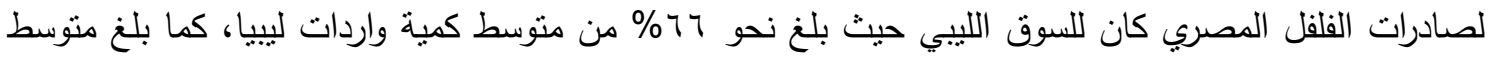

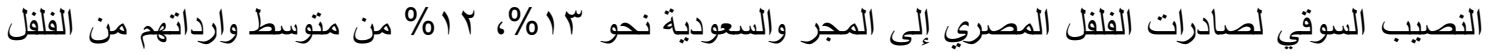
لكلا منهما على الترتيب، في حين بلغ متوسط النصيب السوقي لصادرات الفلفل المصري إلي الكويت، قطر،

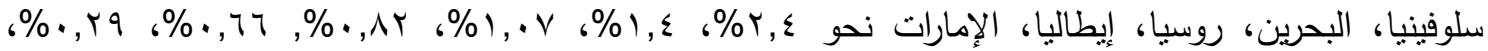
7 ا , • \% من متوسط وارداتهم من الفلفل لكل منهما على الترتيب.

- مؤشر اخترلق السوق لصادرات القلقل المصري في أهم الأسواق العالمية : بلغ مؤشر اختراق السوق للصادرات المصرية من الفلفل المصري للسوق السعودي نحو 9.9\%٪، في حين بلغ مؤشر اختراق السوق في كلا من

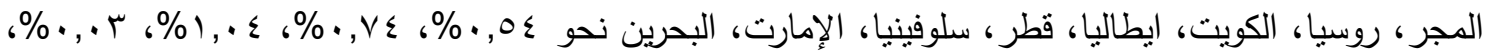

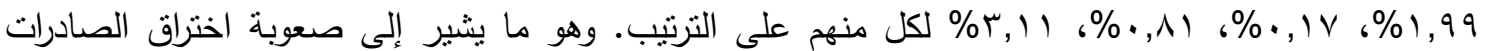
المصرية لهذه الأسواق. 
- مؤثر التنافسية السعرية لصادرات الفلقل المصري في أهم الأسواق العالمية: باستعراض التتافسية السعرية

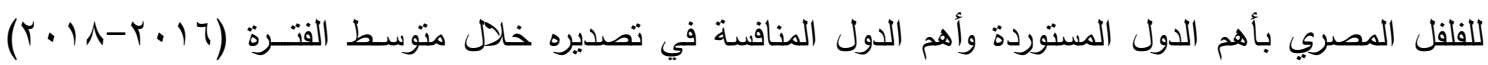
والواردة بالجدول رقم (• (1) تنين أن: باهم الن

جدول رقم (· (1). التنافسية السعرية لصادرات الفلقل المصري في أهم الأسواق العالمية خلال متوسط الفتــرة | - السعر بالدولار / طن

$(r+1 \Lambda-r+17)$

\begin{tabular}{|c|c|c|c|c|c|c|c|}
\hline السعريةة التنافية & التصدير(سيف) & الدافسة & المستوردة & السعرية\%ة التنفية & سعر التصديف) & المنافسة & المستوردة \\
\hline & $1 \leqslant 01,0$ & مصر & \multirow{4}{*}{ قطر } & & $1 \pi 9 \varepsilon, r$ & مصر & \multirow{4}{*}{ السعودية } \\
\hline $1 \cdot r, \Lambda$ & $1 \leqslant \cdot \varepsilon, \wedge$ & الهند & & $1 \leqslant 1, r$ & $9 \wedge 7, \varepsilon$ & الأردن & \\
\hline$r V \cdot, T$ & ors, 9 & الأردن & & $1 \leqslant v$, & $9 \leq \wedge, 7$ & الهند & \\
\hline$\leq \vee 0,1$ & $r \cdot v, \cdot$ & إيران & & $9 \vee$, & $1 \leqslant r V, V$ & بنجلاديش & \\
\hline & Tros, 9 & مصر & \multirow{4}{*}{ سلوفينيا } & & 1787,9 & مصر & \multirow{4}{*}{ المجر } \\
\hline 1790,1 & $V \varepsilon$, & إسر ائيل & & $q r, q$ & $\mid \Lambda \cdot \varepsilon, 1$ & ألمانيا & \\
\hline$r(Y) 9,0$ & 07,0 & إيطاليا & & $\varepsilon q \cdot, r$ & $r \leq r$ & صربيا & \\
\hline ovA.,A & 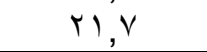 & كرواتيا & & $\wedge, \wedge$ & $19.7 \varepsilon$ & أسبانيا & \\
\hline & $1 \leqslant r \cdot, V$ & مصر & \multirow{4}{*}{ الأمارات } & & 1 IrT,0 & مصر & \multirow{4}{*}{ روسيا } \\
\hline $1 \Lambda \varepsilon, \varepsilon$ & $\checkmark V O, Y$ & الهند & & $\wedge 9, \wedge$ & $1 \leqslant V 7,0$ & إسرائيل & \\
\hline$\varepsilon 01,7$ & rIr, & إيران & & 1 ro, 9 & $1.0 \leqslant$, & أصين & \\
\hline ror,A & 077. & الأردن & & $\varepsilon q \uparrow, \wedge$ & YฯA, T & بيلاروسيا & \\
\hline \multirow{3}{*}{$\varepsilon \cdot v, \wedge$} & $17.7,1 \leq$ & مصر & \multirow{8}{*}{ البحرين } & & lOVY, 2 & مصر & \multirow{4}{*}{ إيطاليا } \\
\hline & & السعه دية & & 111,1 & r'וז'וזו & أسبانيا & \\
\hline & एव & السعودياة & & IrA, & ITrA, 9 & فرنستا & \\
\hline$\leqslant 10, \wedge$ & $r \wedge \uparrow, r$ & الأردن & & $\Delta r, q$ & 1199, & هولندا & \\
\hline \multirow{4}{*}{$1 \cdot 7, \varepsilon$} & \multirow{4}{*}{$10.9,1$} & \multirow{4}{*}{ الهند } & & & $107 \mathrm{~V}, 7$ & مصر & \multirow{4}{*}{ الكويت } \\
\hline & & & & rrA, & $\varepsilon V V, q$ & الأردن & \\
\hline & & & & 109,1 & $910, r$ & الهند & \\
\hline & & & & $r V v, \varepsilon$ & $\varepsilon 10, r$ & السعودية & \\
\hline
\end{tabular}

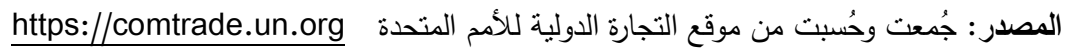

-السوق السعودي: يتمتع الفلفل المصري بميزة نتافسية سعرية بالسوق السعودي مقارنة بمنوسط سعر تصدير بنجلاديش حيث يقل متوسط سعر تصدير الفلفل المصري عن متوسط سعر تصدير الفلفل البنجلاديثي بنسبة

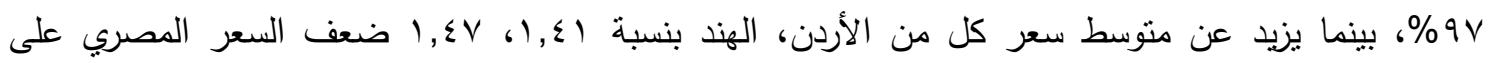

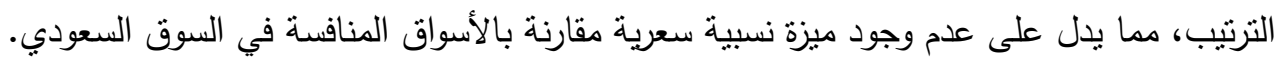
السوق المجري: يتمنع الفلفل المصري بميزة تتافسية سعرية بالسوق المجري مقارنة بمتوسط سعر كلا من ألمانبا،

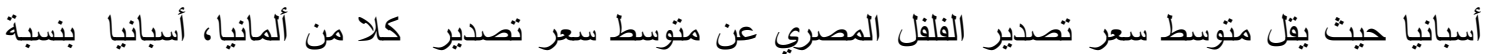

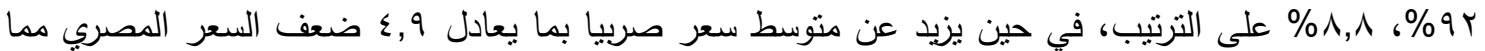
يدل على عدم وجود ميزة نسبية سعرية مقارنة بهذا السوق. -السوق الروسي: يتمتع الفلفل المصري بميزة تتافسية سعرية بالسوق الروسي مقارنة بمتوسط سعر تصدير إسرائيل حيث يقل منوسط سعر تصدير الفلفل المصري عن متوسط سعر تصدير الفلفل الإسرائيلي بنسبة ^,^^\%٪، بينما 
يزيد عن منوسط سعر كل من الصين، بيلاروسيا بما يعادل هץ, ا، بو,؛ ضعف السعر المصري على التزتيب، مما يدل على عدم وجود ميزة نسبية سعرية مقارنة بهذه الأسواق المنافسة.

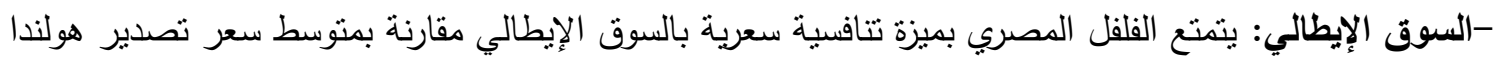

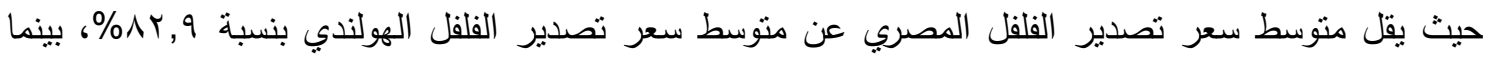

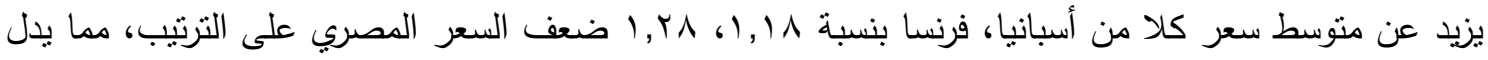
على عدم وجود ميزة نسبية سعرية مقارنة بهذه الأسواق المنافسة في السوق الإيطالي.

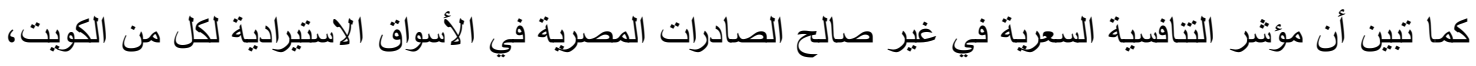

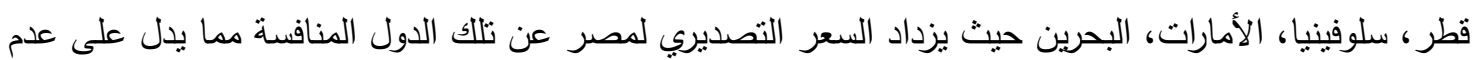
وجود ميزة نسبية سعرية مقارنة بأسعار الدول المنافسة.

التوصية توصى الدراسة بالتوسع في زراعة وإنتاج الفلفل في مصر سواء في الحقول المكثوفة أو المحمية، مع التوسع في الإنتاج المحمي لما يتميز من قدرات إنتاجية عالية، وزراعة نظيفة خالية من المبيدات والتلوث وتنفق مع معايير

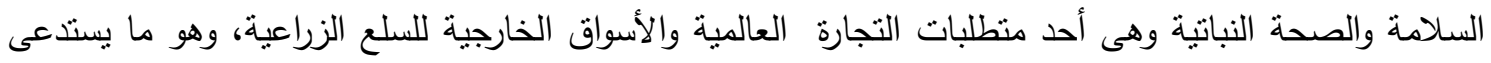

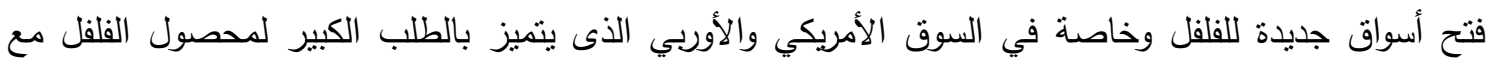
الأخذ في الاعتبار أسعار الدول المنافسة لتصدير الفلفل.

\section{المراجع}

الجهاز المركزى للتعبئة العامة والاحصاء، (1 ا ـ Y). النشرة السنوية للتجارة الخارجية. الجهاز المركزى للتعبئة العامة والاحصاء، (1 ( + r). احصاءات التجارة الخارجية للصادرات والوردات الزراعية.

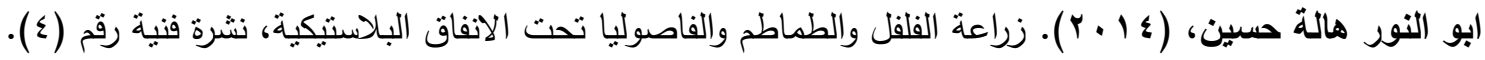
قسم بحوث التسويق الزراعى، (1) (Y). الوضع الراهن للزراعة المحمية في مصر، معهد بحوث الاقتصاد الزراعى، مركز البحوث الزراعية. زكي مراد، وآخرون، (؟ ا + ب). التجارة الخارجية البينية الزراعية العربية (الوضع الراهن وآفاق المستقبل)، مجلة

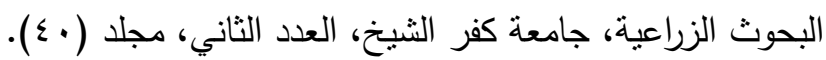

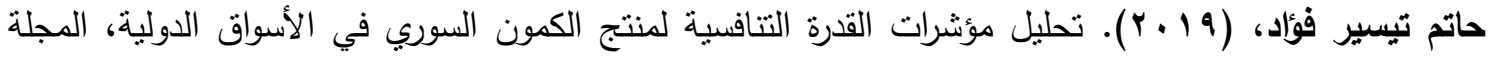
السورية للبحوث الزراعية. عبد الرحمن السيا السيد جاد، (9 ج . ب). الطلب على الموالح المصرية في الأسواق العالمية بإستخدام أساليب التتبؤ رسالة دكتوراه - قسم الإقتصاد الزراعي - كلية الزراعة - جامعة عين شمس.

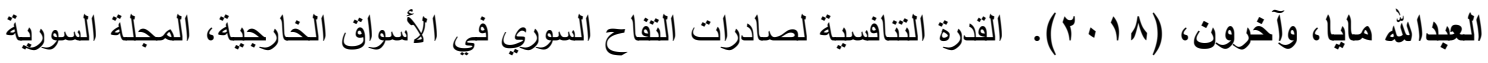

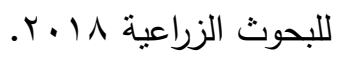

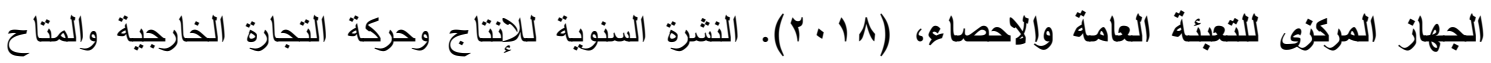
لـاستهلاك من السلع الغذائية. 


$$
\begin{aligned}
& \text { وزارة الزراعة ( • ( + ). المركز الوطنى للبحوث الزراعية ونقل التكنولوجيا، الدليل الفني لمحصول الفلفل، الاردن. } \\
& \text { وزارة الزراعة قطاع الثئون الاقتصادية، الإدارة المركزية للاقتصاد الزراعى، نشرة الاحصاءات الزراعية، القاهرة. } \\
& \text { وزارة الزراعة. قطاع الثئون الاقتصادية، الإدارة المركزية للاقتصاد الزراعى، نشرة الميزان الغذائى، القاهرة. }
\end{aligned}
$$

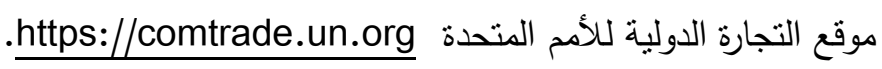

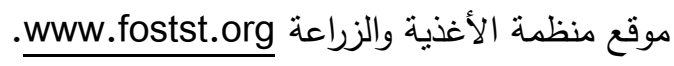

\title{
Summary
}

\section{An Economic Study for the Foreign Trade of Egyptian Pepper}

\author{
Engy Ahmed Teimaa , Eman Abd ELaziz Ibrahim \\ Researcher, Agricultural Economics Research Institute, A.R.C
}

\begin{abstract}
The research aimed to identify the current situation of the production and foreign trade of Egyptian pepper, the research relied on achieving its objectives on both the use of the descriptive and standard analytical method.
\end{abstract}

The research reached a set of results. the most important of which are:

1 - The total production of pepper ranged between a minimum of about 481 thousand tons in 2004 and a maximum of about 825.3 thousand tons in 2009 with an annual average of 675.15 thousand tons. The total production of pepper crop in Egypt has taken an increasing and significant with an annual growth rate of $2 \%$ with an annual change of 13.5 thousand tons. While the statistical significance of the total cultivated area in Egypt did not prove at different levels of significance. as a study of the average per capita pepper showed that it ranged between a minimum of about 6.71 kglyears in 2004 and a maximum of about $10.57 \mathrm{kglyears}$ in 2009 with an annual average of about $8.5 \mathrm{kglyears}$.

2- The results show that the United States of America. Germany. the United Kingdom. France are ranked first from the fourth place for countries importing pepper with a percentage of about $34 \% .12 .16 \% .7 .16 \%$ and $5.07 \%$ of the total world imported pepper. which amounts to 3.29 million tons.

3- It became clear from the geographical distribution of the Egyptian exports of peppers that each of Saudi Arabia, Hungary, Libya, Russian and Kuwait with a concentration factor that reached about 59.68\%. 39.08\%, 35.92\%, $22.71 \%$ and $8.75 \%$. While the geographical concentration coefficients for Italy, Qatar, Slovenia, the Emirates and Bahrain are around sixth to tenth $7.32 \%, 3.08 \%$ $3.77 \%, 1.21 \%, 1.96 \%$ each, respectively. And the amount of Egyptian pepper exports to these tenth countries amounted to about 8.38 thousand tons, representing about $95.8 \%$.

4- Libya is the largest average market share for Egyptian pepper exports. as it reached about $66 \%$ of the average Libyan imports. as the average share of Egyptian pepper to Hungary and Saudi Arabia reached about 13\%. 12\% of their average imports of pepper for each of them. Ranking as the market penetration index for Egyptian exports of Egyptian pepper to the Saudi market amounted to about $9.97 \%$. while the market penetration index for the market in Hungary. Russia. Kuwait. Italy. Qatar. Slovenia. UAE and Bahrain reached about $0.54 \%$, $0.74 \%, 1.04 \%, 0.03 \%, 1.99 \%, 0.17 \%, 0.81 \%, 3.11 \%$ each of them, respectively. 
This indicates the difficulty of penetrating Egyptian exports to these markets. Egyptian pepper has a competitive advantage in the Saudi market compared to the average export price of Bangladesh. and in the Hungarian market compared to the average export price of Germany. Spain. and the Russian market compared to the average export price of Israel. as it was found that there is no comparative price advantage for Kuwait, Qatar, Slovenia, The Emirates, Bahrain compared to the prices of the competing countries.

Key words: Agriculture Foreign Trade - Egyptian Pepper - Competitiveness indicators, Market share, Geographical concentration factor, Price competitiveness. 